\title{
Behavioral correlates of semi-zygodactyly in Ospreys (Pandion haliaetus) based on analysis of internet images
}

\author{
Diego Sustaita ${ }^{\text {Corresp., }}{ }^{1}$ ， Yuri Gloumakov ${ }^{2}$ ， Leah R Tsang ${ }^{3,4}$ ， Aaron M Dollar ${ }^{2}$ \\ ${ }^{1}$ Department of Biological Sciences, California State University, San Marcos, San Marcos, California, United States \\ 2 Department of Mechanical Engineering and Materials Science, Yale University, New Haven, CT, United States \\ 3 Department of Zoology, Environmental and Rural Sciences, University of New England, Armidale, New South Wales, Australia \\ 4 Ornithology Collection, Australian Museum Research Institute, Sydney, New South Wales, Australia \\ Corresponding Author: Diego Sustaita \\ Email address: dsustaita@csusm.edu
}

Ospreys are renowned for their fishing abilities, which have largely been attributed to their specialized talon morphology and semi-zygodactyly-the ability to rotate the fourth toe to accompany the first toe in opposition of toes II and III. Anecdotal observations indicate that zygodactyly in Ospreys is associated with prey capture, although to our knowledge this has not been rigorously tested. As a first pass toward understanding the functional significance of semi-zygodactyly in Ospreys, we scoured the internet for images of Osprey feet in a variety of circumstances. From these we cross-tabulated the number of times each of three toe configurations (anisodactylous, zygodactylous, and an intermediate condition between these) was associated with different grasping scenarios (e.g., grasping prey or perched), contact conditions (e.g., fish, other objects, or substrate), object sizes (relative to foot size), and grasping behaviors (e.g., using one or both feet). Our analysis confirms an association between zygodactyly and grasping behavior; the odds that an osprey exhibited zygodactyly while grasping objects in flight were 5.7 times greater than whilst perched. Furthermore, the odds of zygodactyly during single-foot grasps were 4.1 times greater when pictured grasping fish compared to other objects. This suggests a functional association between predatory behavior and zygodactyly and has implications for the selective role of predatory performance in the evolution of zygodactyly more generally. 
1 Title: Behavioral correlates of semi-zygodactyly in Ospreys (Pandion haliaetus) based on

2 analysis of internet images

3

4 Authors: Diego Sustaita ${ }^{1}$, Yuri Gloumakov ${ }^{2}$, Leah R. Tsang ${ }^{3,4}$, Aaron M. Dollar $^{2}$

$5{ }^{1}$ Department of Biological Sciences, California State University, San Marcos, 333 S. Twin Oaks

6 Valley Rd., San Marcos, CA 92096

$7 \quad{ }^{2}$ Department of Mechanical Engineering and Materials Science, Yale University, 9 Hillhouse

8 Ave, New Haven, CT 06511

$9{ }^{3}$ Department of Zoology, Environmental and Rural Sciences, University of New England,

10 Armidale, NSW 2350

$11{ }^{4}$ Ornithology Collection, Australian Museum Research Institute, 1 William Street, Sydney, NSW

$12 \quad 2010$

13

14 Corresponding author: Diego Sustaita (dsustaita@csusm.edu)

\section{Abstract}

Ospreys are renowned for their fishing abilities, which have largely been attributed to their specialized talon morphology and semi-zygodactyly-the ability to rotate the fourth toe to accompany the first toe in opposition of toes II and III. Anecdotal observations indicate that zygodactyly in Ospreys is associated with prey capture, although to our knowledge this has not been rigorously tested. As a first pass toward understanding the functional significance of semizygodactyly in Ospreys, we scoured the internet for images of Osprey feet in a variety of circumstances. From these we cross-tabulated the number of times each of three toe 
24 configurations (anisodactylous, zygodactylous, and an intermediate condition between these)

25

was associated with different grasping scenarios (e.g., grasping prey or perched), contact

conditions (e.g., fish, other objects, or substrate), object sizes (relative to foot size), and grasping behaviors (e.g., using one or both feet). Our analysis confirms an association between zygodactyly and grasping behavior; the odds that an osprey exhibited zygodactyly while grasping objects in flight were 5.7 times greater than whilst perched. Furthermore, the odds of zygodactyly during single-foot grasps were 4.1 times greater when pictured grasping fish compared to other objects. This suggests a functional association between predatory behavior and zygodactyly and has implications for the selective role of predatory performance in the evolution of zygodactyly more generally.

\section{INTRODUCTION}

Ospreys (Accipitriformes: Pandionidae:Pandion haliaetus) feed virtually exclusively on fish (accounting for $\sim 99 \%$ of their diet) that they take from the water (Bierregaard et al., 2016). They are able to achieve substantial prey-capture success rates for a predator (up to $82 \%$; Bierregaard et al., 2016), despite the difficulties inherent when plunge-diving feet-first to capture fish. This ability is afforded by their unique pedal anatomy, compared to other birds of prey. Among these attributes is the ability to rotate the fourth toe (digit IV) antero-posteriorly, and toggle between anisodactyl (digits II-IV face anteriorly; digit I posteriorly) and zygodactyl (digits II and III face anteriorly; digits I and IV face posteriorly) toe arrangements (Shufeldt, 1909; Jollie, 1976, 1977; Raikow, 1985; Polson, 1993; Ramos and Walker, 1998; Tsang and McDonald, 2018) (Fig. 1F). The ability to facultatively shift from anisodactyly to zygodactyly (i.e., semi-zygodactyly; Raikow, 1985) is thought to enhance their extreme grasping capabilities. For instance, previous 
47 researchers have proposed that the facultative zygodactyl arrangement in predatory birds, such as

48 owls (Strigiformes) and Black-shouldered Kites (Accipitriformes: Accipitridae: Elanus axillaris)

49 (Tsang, 2012), provides advantages for distributing the toes (and prey-contact surface area) more

50 symmetrically (Payne, 1962; Goslow, 1972), as well as for generating greater grip strength

51 (Ward et al., 2002; Einoder and Richardson, 2007). Both of these advantages ostensibly pertain

52 to the Osprey, which grasps evasive, slippery fish from above by plunge-diving to capture prey

53 about half a meter below the surface of the water (Polson, 1993).

That Ospreys can reverse their fourth toe to assume a zygodactyl foot configuration is fairly well

known (e.g., Johnsgard, 1990; Olsen, 1995; Ferguson-Lees \& Christie, 2001; Bierregaard et al., 2016). However, it is not abundantly clear specifically when and how they employ one toe configuration over the other. Casual observations of ospreys captured in photographs reveal that the zygodactyl configuration is often assumed during perching as well as when clutching fish. Thus, the extent to which Ospreys preferentially use zygodactyly for grasping prey, although perfectly reasonable, is not explicitly clear. Furthermore, it is unclear specifically how the change in toe configuration is controlled. Ospreys possess gross anatomical peculiarities that are presumed to be associated with semi-zygodactyly. These include a relatively long digit IV that is semi-reversible, the absence of a membrane between digits III and IV (Tsang, 2012), and claws of near equal length across all toes (Hudson, 1948; Jollie, 1976, 1977). In regards to the underlying bones, the inner trochlea of the distal tarsometatarsus is comparatively more developed than in other accipitriforms, which might afford the second digit a relatively greater range of motion, and the shape of the outer trochlea seems to reflect the "wide lateral movement" exhibited by digit IV (Jollie, 1976, 1977). Finally, their comparatively well-developed M. 
70

71

72

lumbricales and M. abductor digiti IV muscles (Hudson, 1948) reflect their keen abilities to abduct and reverse digit IV, unlike other raptors. However, the extent to which Ospreys are able to reposition digit IV voluntarily, or if such repositioning is mechanistically coupled with other hindlimb or digital movements, is unclear.

As part of a larger project aimed at understanding the anatomy, control, and functional significance of semi-zygodactyly in Ospreys, we first set out to examine the behavioral correlates of semi-zygodactyly. We approached this by quantifying foot use behaviors captured in digital images and videos publicly available on the internet, using a methodology similar to Allen et al.'s (2018) image-based study of foot lateralization in Ospreys, although derived independently. We used data gleaned from these images specifically to test for associations among toe configurations, grasping scenario, and object size (Fig. 1). Following conventional wisdom, we predicted that Ospreys photographed clutching fish were more likely to display a zygodactyl $(2 \times 2)$ toe configuration. Furthermore, under the assumption that zygodactyly enhances grip force or the probability of prey contact (cited above), we anticipated that larger object (prey) sizes, (but not necessarily perching substrates), would also elicit a $2 \times 2$ toe configuration.

\section{MATERials AND Methods}

We searched the World-Wide Web (predominantly Google Images [English]) for photographs of Ospreys interacting with prey or various substrates, using the following search terms: "osprey," "Pandion haliaetus," and combinations of the previous two terms with "clutching," "grasping," "nest," "fish," and "photos." We then moved on to searching both personal and professional websites, and then videos (where we took screenshots of appropriate footage). Finally, we moved 
93 on to different languages of Google and repeated the above. Two observers independently scored

94 each foot of each Osprey in every image for the characteristics described below and in Table 1.

95 A third independent observer served as a "moderator," by compiling the scores of the other two

96 observers and resolving any disagreements. The three observers rotated among tasks, such that

97 each one served as a moderator for one component of the data set or another. We screened the

98 data set for duplicated image file names to avoid scoring the same individual Osprey foot twice.

99 We also ordered images by size and dimension to guard against the possibility that duplicated

100 images were uploaded with different file names. Nevertheless, we cannot exclude the possibility

101 that the same individual Ospreys might have appeared in more than one distinct image. The

102 inadvertent inclusion of duplicated images and/or individual Osprey feet (i.e., pseudoreplication)

103 would certainly inflate type I error rates. However, we have no reason to suspect any biases in

104 the likelihood of duplicated images with respect to toe configuration and grasping condition, in

105 which case the magnitudes (if not the significance levels) of the relationships among variables

106 should remain relatively unaffected.

107

108

Each Osprey pictured in an image constituted a "subject," and each foot pictured was a replicate

in the analyses. We used generalized estimating equations (a repeated-measures form of logistic

regression; SPSS, 2013), with image identity included as a subject variable, and foot identity

111 (left or right) included as a within-subjects variable, for which we specified an unstructured

112 correlation matrix. We treated toe configuration as an ordinal (logistic) response variable ranging

113 between $1(=3 \times 1)$ and $3(=2 \times 2)$, in which $2(=2.5 \times 1.5)$ constituted an intermediate

114 configuration analogous to Bock and Miller's (1959) “ectropodactyl” foot type (Fig. 1B, C, F).

115 We performed two series of analyses: one overall test to examine the effects of relative "object 
116 size" (ordinal variable ranging 0 [no object] to 4 [extra-large]; Table 1) and "grasping scenario"

117 ( 0 = nothing in feet, $\mathrm{P}=$ perched on substrate, $\mathrm{G}=$ grasping an object $)$, as well as their

118 interaction. Although we were not specifically interested in the effects of foot identity (left or

119 right), we performed an additional test including "foot identity" as a fixed effect to screen for

120 any footedness biases (e.g., Allen et al., 2018). We then followed this analysis with a more

121 refined test on data including only cases of contact between foot and object or substrate. For this

122 test, we included an additional nested effect of "contact condition" $(\mathrm{F}=$ fish, $\mathrm{O}=$ other object, $\mathrm{T}$

$123=$ tree, $\mathrm{S}=$ other substrate; Table 1) within grasping scenario (P vs. G), to determine whether the

124 general types of objects or substrates grasped have any further effects on toe configuration within

125 each of the two main grasping scenarios. We also added an additional variable, "footing,"

126 indicating whether grasping was performed with one or both feet. For both sets of analyses, we

127 began with full models (main effects and interactions) and successively removed non-significant

128 interactions (by order of decreasing $P$-value) to obtain the most parsimonious final models.

129 Significance was based on the Type III sums of squares, and an $\alpha=0.05$.

\section{RESULTS}

132 The 1184 images of Osprey grasping behavior that we scored (Supplemental Data S1) fell into

133 five main categories: (1) flying with fish, perching (2) with and (3) without fish, (4) nest-

134 building, and (5) pre-contact with prey or substrate. Of these, obscured visibility of the feet and 135 casewise deletions from one or more missing variables resulted in 1123 Osprey images of $n=$ 1361882 feet, both in contact with objects and not, entered into the analysis. Overall, there was no 137 significant interaction between object size and grasping scenario on toe configuration (Type III 138 Wald Chi-square $\left(\chi^{2}\right)$ test of model effects $\left.=4.34, \mathrm{df}=2, P=0.114\right)$. The effect of grasping 
139 scenario remained significant $\left(\chi^{2}=198.61, \mathrm{df}=1, P<0.0001\right)$, and the effect object size

140 remained non-significant $\left(\chi^{2}=0.457, \mathrm{df}=3, P=0.928\right)$, after removing the non-significant

141 interaction term from the model. The parameter estimates ( $B$; Table 2$)$ revealed that the

142 probability of zygodactyly significantly increased for the 'object grasping' and 'nothing in foot'

143 scenarios, compared to the 'perching' scenario (Fig. 2). In particular, the odds that an osprey

144 exhibited a zygodactyl toe configuration during flight were 5.7 times greater when pictured

145 grasping objects, and 2.6 times greater when grasping nothing, than whilst perched. There was

146 no significant effect of foot identity (confirming of the lack of foot lateralization in Ospreys

147 found by Allen et al. [2018]), nor any interaction with objects size or grasping scenario, on toe

148 configuration (Supplemental Table S1).

150 When considering object-contact cases only ( $n=1503$ feet from 995 images), all main effects

151 and interactions were significant (Table 3). Both interaction effects involving footing and object

152 size reflect variation in responses between contact conditions within each perching and grasping

153 scenarios (Fig. 3). In the former case, the interaction was due primarily to an increase in the

154 probability of zygodactyly from dual- to single-foot grasping for fish, relative to the "other

155 substrate" reference contact condition of perching $(B=0.882 \pm 0.378, \mathrm{df}=1, P=0.019, \operatorname{Exp}(B)$

$156=2.42[1.15-5.07,95 \% \mathrm{CI}])$. The latter interaction was due to two marginally non-significant $(P$

$157=0.071-0.072)$ effects: a decrease in the probability of zygodactyly for small object sizes relative

158 to large when grasping fish compared to the "other substrate" reference condition of perching,

159 and an increase in the probability of zygodactyly for medium object sizes relative to large, when

160 perched in trees compared to "other substrates". However, because these parameters were not 
161 significant, we felt justified in excluding the object size $\times$ contact condition within grasping

162 scenario interaction effect in subsequent analyses (below).

163

164 In the subsequent model, all effects remained significant, with the exception of object size (Table

165 3). Because the effect of contact condition within grasping scenario depended upon whether or

166 not the grasp was single- or dual-footed, we generated new models for dual-footed $(n=962)$ and

167 single-footed $(n=541)$ grasps, separately (Fig. 3). In both models the main effect of grasping

168 scenario was significant (Table 3), such that the odds of zygodactyl grasps were 2.8 and 6.4

169 times greater during flying than perching (Table 4). Furthermore, there was a significant effect of

170 contact condition within grasping scenario for single-footed grasps, but not for bi-axial grasps

171 (Table 3). For the former, the probability of zygodactyly was significantly greater for the fish,

172 compared to the "other object" contact condition, as well as for the tree, compared to the "other

173 substrate" contact condition (Table 4).

174

175 DiscussiON

176 We analyzed grasping behavior of Ospreys from 1184 web images and videos of Ospreys in

177 various states of using their feet. Our results support predictions from casual observations,

178 photographs, and anecdotal reports from the literature: that Ospreys tend to employ a

179 zygodactylous foot configuration when grasping objects, and in particular when gripping fish.

180 This suggests a functional association between predatory behavior and zygodactyly and has

181 implications for the selective role of predatory performance in the evolution of zygodactyly more

182 generally. Notably, the use of a zygodactylous configuration during single-foot grasps of fish

183 (e.g., Fig. 3) strongly suggests that this toe configuration affords a performance advantage under 
184 the most challenging grasping conditions. Along these lines, however, it seems odd that object

185 size was ostensibly unrelated to zygodactyly (e.g., Fig. 2), with a (non-significant) tendency for

186 zygodactyl toe configurations to be pictured with smaller object sizes. On biomechanical

187 grounds, very large and very small objects (relative to grasper size) pose greater challenges for

188 grasping (e.g., Seo \& Armstrong, 2008; Irwin \& Radwin, 2008; Fok \& Chou, 2010). Perhaps this

189 is explained by the potential benefits of the multiarticular nature of their digital flexion

190 mechanism (Backus et al., 2015), which might afford the ability to grasp a wide range of object

191 sizes regardless of toe configuration (Dollar \& Howe, 2011).

192

193 Embryological evidence supports developmental mechanisms as the primary drivers of toe 194 configuration across taxa (Botelho et al., 2014, 2015). Semi-zygodactyly appears in four avian 195 clades: Ospreys, turacos, the common ancestor of owls and mousebirds (Botelho et al., 2015), 196 and in ancestral accipitrid kites (Tsang 2012), most likely having arisen independently in some 197 of these lineages (Ksepka et al., 2017). In most of these cases, semi-zygodactyly occurs in 198 groups related to fully-zygodactylous clades, suggesting semi-zygodactyly as a potential 199 intermediate, ancestral condition (Botelho et al., 2015; Ksepka et al., 2017). However, semizygodactyl Ospreys (Pandionidae) are nested well within the predominantly anisodactylous Accipitriformes (Hackett et al., 2008; Yuri et al., 2013; Jarvis et al., 2014; Prum et al., 2015), which, coupled with their extreme piscivorous specialization, suggests a possible adaptive role

203 for semi-zygodactyly in this group. Furthermore, a recent analysis of the pedal flexibility of 204 Australian raptors, including the Osprey, has indicated that diurnal raptors do indeed possess a wide range of angle divarication of digits (i.e., the degree to which toes are splayed out from one 206 another) as a group (Tsang \& McDonald, 2018). The Osprey exceeded the maximum digit angle 
207 divarication of digit IV (the digit that enables semi-zygodactyl grasping) of other anisodactylous

208 raptors, achieving wider digit IV angle divarication results that overlapped with the digit IV

209 angle divarications of the nocturnal owls. This degree of convergence between Ospreys and owls

210 lends further support to possible ecological, adaptive, origins of semi-zygodactyly, because

211 Ospreys and owls feed mostly on prey that can be difficult to capture (e.g., Ospreys plunge-

212 diving for slippery fish and owls nocturnally hunting fast-moving small mammals). Both groups

213 rely mostly on stealth to snatch their prey unawares from under the cover of water or darkness,

214 respectively. However, both groups also possess morphological and behavioral modifications

215 presumed to meet the potential added challenges of their prey. For example, both groups lack a

216 stretch of skin between digits III and IV typical of other raptors, which facilitates a wider lateral

217 movement of digit IV. Furthermore, previous researchers have cited the relatively strong

218 gripping forces of owls compared to diurnal raptors, as a mechanism for overcoming the

219 relatively greater difficulties they experience hunting nocturnally (Marti, 1974; Ward et al.,

220 2002; Einoder \& Richardson, 2007).

221

222 However, there are other taxa that are semi-zygodactylous (e.g., mousebirds and turacos),

223 zygodactylous (e.g., parrots, woodpeckers, and roadrunners), and heterodactylous (trogons) that

224 do not capture prey with their feet as do Ospreys and owls, and there are several other

225 anisodactylous species that do (e.g., falconiforms and most accipitriforms) (Raikow, 1985).

226 Although functional, adaptive arguments have been made for some of these (e.g., for climbing or

227 manipulating food with the toes; Bock \& Miller, 1959; Berman \& Raikow, 1982), definitive

228 conclusions await more comprehensive, phylogenetically-informed analysis. Nevertheless, 
229 developmental mechanisms and ecological factors are not mutually exclusive, and it is likely that

230 different combinations of these factors influence the evolution of semi-zygodactyly across taxa.

232 The ability to transition between toe configurations is a feat of which very few species are

233

234

235

236

237

238

239

240

241

242

243

244

245

246

247

248

249

250

251

capable. We present quantitative data linking prey capture behavior with zygodactyly in Ospreys.

Nevertheless, the extent to which semi- or full-zygodactyly provides a distinct performance advantage for grasping has yet to be explicitly tested. Bartosik (2009) suggested that the flexibility afforded by the ability to reverse the outer toe helps Ospreys optimize their grasps on the lateral sides of fish so as to avoid contact with their sharp dorsal spines. Thus, further work is required, supported by consistent field observations of reliably located individuals at close range, to facilitate further study of this unique behavior. Citizen science potentially has much to offer in this regard, via nest cams or automated cameras positioned near prime foraging grounds (Bierregaard et al., 2014). Another important avenue of inquiry currently underway is to uncover precisely how rotation of the outer toe is controlled; i.e., whether it is driven entirely by the action of M. abductor digit IV, or through joint coupling mechanisms facilitated by the morphology of the tarsometatarso-phalangeal joint and other multiarticular digital tendons.

\section{CONCLUSIONS}

From our analysis of web images, we found that semi-zygodactylous Ospreys are pictured using three predominant toe configurations: anisodactylous, zygodactylous, and an intermedite condition we labeled " $2.5 \times 1.5$ ". Our generalized estimating equation models confirmed the oftcited association between zygodactyly and grasping behavior in general; the odds that an osprey exhibited zygodactyly while pictured grasping objects in flight were 5.7 times greater than whilst 
252 perched. Contrary to our expectations, zygodactyly was unrelated to object size, but the odds of

253 observing zygodactyly in single-foot grasps were 4.1 times greater with fish compared to other

254 objects. This suggests a functional association between predatory behavior and zygodactyly, and

255 ultimately has implications for the selective role of predatory performance in the evolution of 256 zygodactyly.

257

258 ACKNOWLEDGEMENTS

259 We kindly thank Hans Kassier, Chad Small, and Jon Simmonds for their hard work and

260 countless hours accessing and scoring digital web images of Ospreys. We are very grateful to

261 Julieta Carril, Richard O. Bierregaard, and another anonymous reviewer, whose constructive

262 comments greatly improved the final version of this paper.

263

264 REFERENCES

265 Allen LL, Morrison KL, Scott WAE, Shinn S, Haltiner AM, Doherty MJ. 2018. Differences between stance and foot preference evident in Osprey (Pandion haliaetus) fish holding during movement. Brain and Behavior e01126 DOI: 10.1002/brb3.1126

268

269

270

271

272

273
Backus SB, Sustaita D, Odhner LU, Dollar AM. 2015. Mechanical analysis of avian feet: multiarticular muscles in grasping and perching. Royal Society Open Science 2:140350 DOI 10.1098/rsos.140350.

Bartosik MB. 2009. Osprey (Pandion haliaetus): notes on unknown and poorly studied behaviors. Bulletin of the Texas Ornithological Society 42:18-36 http://www.texasbirds.org/publications.php 
274 Berman SL, Raikow RJ. 1982. The hindlimb musculature of the mousebirds (Coliiformes). Auk

275

276

277

278

279

280

281

282

283

284

285

286

287

288

289

290

291

292

293

294 99:41-57 DOI: 10.2307/4086020.

Bierregaard RO, Poole AF, Martell MS, Pyle P, Patten MA. 2016. Osprey (Pandion haliaetus), version 2.0. In The Birds of North America (P. G. Rodewald, Editor). Cornell Lab of Ornithology, Ithaca, NY, USA. https://doi.org/10.2173/bna.683 (last accessed 23 August 2018).

Bierregaard RO, Poole AF, Washburn BE. 2014. Ospreys (Pandion haliaetus) in the $21^{\text {st }}$ century: populations, migration, management, and research priorities. Journal of Raptor Research 48: 301-308 DOI 10.3356/0892-1016-48.4.301.

Bock WJ, Miller WD. 1959. The Scansorial Foot of the Woodpeckers, with Comments on the Evolution of Perching and Climbing Feet in Birds. American Museum Novitates 1931:145.

Botelho JF, Smith-Paredes D, Nuñez-Leon D, Soto-Acuña S, Vargas AO. 2014. The developmental origin of zygodactyl feet and its possible loss in the evolution of Passeriformes. Proceedings of the Royal Society B 281: 20140765 DOI 10.1098/rspb.2014.0765.

Botelho JF, Smith-Paredes D, Vargas AO. 2015. Altriciality and the Evolution of Toe Orientation in Birds. Evolutionary Biology 42:502-510 DOI 10.1007/s11692-015-9334-7.

Dollar AM, Howe RD. 2011. Joint coupling design of underactuated hands for unstructured environments. International Journal of Robotics Research 30:1157-116 DOI $10.1177 / 0278364911401441$. 
295 Einoder LD, Richardson AMM. 2007. Aspects of the hindlimb morphology of some Australian

296 birds of prey: a comparative and quantitative study. Auk 124:773-788 DOI

297 10.1642/0004-8038(2007)124[773:AOTHMO]2.0.CO;2.

298 Ferguson-Lees J, Christie DA. 2001. Raptors of the world. Christopher Helm, London.

299 Fok KS, Chou SM. 2010. Development of a finger biomechanical model and its considerations. $300 \quad$ Journal of Biomechanics 43:701-713 DOI 10.1016/j.jbiomech.2009.10.020.

301 Goslow GE Jr. 1972. Adaptive mechanisms of the raptor pelvic limb. Auk 89:47-64.

302 Hackett SJ, Kimball RT, Reddy S, Bowie RCK, Braun EL, Braun MJ, Chojnowski JL, Cox WA, 303 Han KL, Harshman J, Huddleston CJ, et al. 2008. A phylogenomic study of birds reveals

305

306

307 308 their evolutionary history. Science 320:1763-1768 DOI 10.1126/science.1157704.

Hudson GE. 1948. Studies on the Muscles of the Pelvic Appendage in Birds II: The Heterogeneous Order Falconiformes. The American Midland Naturalist 39:102-127.

Irwin CB, Radwin RG. 2008. A new method for estimating hand internal loads from external force measurements. Ergonomics 51:156-167 DOI 10.1080/00140130701526408.

Jarvis ED, Mirarab S, Aberer AJ, Li B, Houde P, Li C, Ho SYW, Faircloth BC, Nabholz B, Howard JT, Suh A, et al. 2014. Whole-genome analyses resolve early branches in the tree of life of modern birds. Science 346:1320-1331 DOI 10.1126/science.1253451.

Johnsgard P. 1990. Eagles, Hawks and Falcons of North America. Smithsonian Institutional Press, Washington D.C., USA.

Jollie MT. 1976. A contribution to the morphology and phylogeny of the Falconiformes, part 1. Evolutionary Theory 1:285-298.

Jollie MT. 1977. A contribution to the morphology and phylogeny of the Falconiformes, parts 23. Evolutionary Theory 2:115-300. 
318 Ksepka DT, Stidham TA, Williamson TE. 2017. Early Paleocene landbird supports rapid

319 phylogenetic and morphological diversification of crown birds after the K-Pg mass extinction. Proc Natl Acad Sci U S A 114:8047-8052 DOI: 10.1073/pnas.1700188114.

321

322

323

324

325

326

327

328

329

330

331

332

333

334

335

336

337

338

339

Marti CD. 1974. Feeding ecology of four sympatric owls. Condor 76: 45-61 DOI $10.2307 / 1365983$

Olsen P. 1995. Australian birds of prey: the biology and ecology of raptors, UNSW Press, Sydney.

Payne RS. 1962. The acoustical location of prey by the Barn Owl (Tyto alba). Ph.D. Dissertation, Cornell University, Ithaca, New York.

Polson JR. 1993. Weapons to cradle: Birds put the toe to the task. American Birds: 47:216-219.

Prum RO, Berv JS, Dornburg A, Field DJ, Townsend JP, Lemmon EM, Lemmon AR. 2015. A comprehensive phylogeny of birds (Aves) using targeted next-generation DNA sequencing. Nature 526:569-573 DOI 10.1038/nature15697.

Raikow RJ. 1985. The locomotor system. In: King AS, McLelland J, eds. Form and function in birds, vol. 3. London: Academic Press, 357-147.

Ramos AM, Walker ID. 1998. Raptors--inroads to multifingered grasping. In: International conference on intelligent robots and systems. Victoria BC: IEEE/RSJ, 467-475.

Seo NJ, Armstrong TJ. 2008. Investigation of grip force, normal force, contact area, hand size, and handle size for cylindrical handles. Human Factors 50:734-744 DOI $10.1518 / 001872008 X 354192$.

Shufeldt RW. 1909. Osteology of birds. New York State Museum Bulletin 130:90-91. SPSS. 2013. IBM SPSS Statistics, Version 22. IBM, New York. 
340 Tsang LR. 2012. Facultative zygodactyly in the Black-shouldered Kite Elanus axillaris.

$341 \quad$ Australian Field Ornithology 29:89-92.

342 Tsang LR, McDonald PG. 2018. A comparative study of avian pes morphotypes, and the

343 functional implications of Australian raptor pedal flexibility. Emu-Austral Ornithology.

$344 \quad$ DOI: $10.1080 / 01584197.2018 .1483203$.

345 Ward AB, Weigl PD, Conroy RM. 2002. Functional morphology of raptor hindlimbs:

346 implications for resource partitioning. Auk 119:1052-1063 DOI 10.1642/0004-

347 8038(2002)119[1052:FMORHI]2.0.CO;2.

348 Yuri T, Kimball RT, Harshman J, Bowie RC, Braun MJ, Chojnowski JL, Han KL, Hackett SJ,

349 Huddleston CJ, Moore WS, Reddy S, et al. 2013. Parsimony and model-based analyses of

350 indels in avian nuclear genes reveal congruent and incongruent phylogenetic signals.

351 Biology (Basel) 2:419-444 DOI 10.3390/biology2010419. 


\section{Table $\mathbf{1}$ (on next page)}

List of variables included in the analyses, along with descriptions of each category.

Statistical analyses were designed in such a way as to model the probability of zygodactyly (dependent variable) with each condition. 


\begin{tabular}{ll}
\hline Variable/categories & Description \\
\hline \hline Toe configuration & \\
$1(3 \times 1)$ & $\begin{array}{l}\text { Anisodactyl (digits II-IV directed } \\
\text { cranially, digit I directed } \\
\text { caudally) }\end{array}$ \\
$2(2.5 \times 1)$ & $\begin{array}{l}\text { Transitional; digit IV mid-way } \\
\text { between digits III and I }\end{array}$ \\
$3(2 \times 2)$ & $\begin{array}{l}\text { Zygodactyl (digits II and III } \\
\text { directed cranially, digits I and IV } \\
\text { directed caudally) }\end{array}$
\end{tabular}

Grasping scenario

Additional notes/justification

Treated as an ordinal logistic

response variable

To test how overall grasping behavior affects toe configuration

Free-footed (0) Foot was empty; Osprey may have been landing, taking off, or diving

Grasping object (G) Object visibly clutched by foot; usually during mid-flight

Perching (P) Osprey was apparently motionless, with foot open against substrate

Contact condition

Effect nested within grasping scenario, to determine whether the type of object/structure contacted within each scenario ( $\mathrm{G}$ or $\mathrm{P}$, above) affected toe configuration.

No contact (0) Foot not in contact with anything

Fish (F) $\quad$ Foot enclosed a fish; usually upon leaving the water or in midflight or landing

Other object $(\mathrm{O}) \quad$ Foot enclosed something other than a fish; usually nesting material, occasionally the talons 
of other Ospreys

Tree (T)

Foot was enclosed around a tree branch while Osprey was

perched

Other substrate (S) Foot was in contact with perching substrates other than a tree branch; usually a post, rock, or ground

Object size

$0 \quad$ No object in foot

$1 \quad$ Small/very small: foot encircled between 67 and $\geq 100 \%$ of object "diameter".

2 Medium: foot encircled between 34$66 \%$ of object "diameter".

3 Large: foot encircled $33 \%$ of object or less of object "diameter".

4 Extra-large: foot did not really "wrap" around the object at all (e.g. ground, nest surface).

Foot identity Left or right foot scored

Footing Whether object was grasped with one (1) or both (2) feet
Trees were distinguished from other perching substrates to account for Ospreys' tendencies to wrap their toes around branches, as opposed to standing flat-footed

Assessed visually, relative to the extent to which toes encircled the object

By "diameter" we refer roughly to the cross-sectional dimension of the grasped object

Included as a within-subjects variable to account for covariation in the responses between feet

Included specifically to test whether single-foot grasps were more apt to exhibit zygodactyly, perhaps to enhance purchase on objects when unaided by the other foot 


\section{Table 2 (on next page)}

Parameter estimates and test statistics from a generalized estimating equation (GEE) model.

Toe configuration (toe code; $1=3 \times 1,2=2.5 \times 1.5,3=2 \times 2$ ) was modeled as a function of grasping scenario (graspscen; free-footed, grasping, perched), object size (objsize; no object [0] - extra-large [4]), and their interaction (graspscen $\times$ objsize), for the complete data set ( $n$ $=1882$ feet [of 1123 Osprey images]). 


\begin{tabular}{|c|c|c|c|c|c|c|c|c|}
\hline \multirow[b]{2}{*}{ Parameter* } & \multirow[b]{2}{*}{ B } & \multirow[b]{2}{*}{$\begin{array}{c}\text { Std. } \\
\text { Error } \\
\end{array}$} & \multicolumn{3}{|c|}{ Hypothesis Test } & \multirow[b]{2}{*}{$\begin{array}{c}\text { Odds ratio } \\
\operatorname{Exp}(B)\end{array}$} & \multicolumn{2}{|c|}{ 95\% CI $\operatorname{Exp}(B)$} \\
\hline & & & $\begin{array}{l}\text { Type III } \\
\text { Wald } \chi^{2} \\
\end{array}$ & df & $\boldsymbol{P}$ & & Lower & Upper \\
\hline Threshold toecode $=1$ & .172 & .2226 & .595 & 1 & 2.440 & 1.187 & .768 & 1.837 \\
\hline toecode $=2$ & .173 & .2236 & 11.942 & 1 & .001 & 2.166 & 1.397 & 3.357 \\
\hline graspscen $=0$ & .963 & .2517 & 14.629 & 1 & .0001 & 2.619 & 1.599 & 4.289 \\
\hline graspscen $=\mathrm{G}$ & 1.739 & .3139 & 30.678 & 1 & $<.0001$ & 5.690 & 3.075 & 10.527 \\
\hline objsize $=1$ & .308 & .2472 & 1.550 & 1 & .213 & 1.360 & .838 & 2.208 \\
\hline objsize $=2$ & .046 & .2533 & .033 & 1 & .856 & 1.047 & .637 & 1.720 \\
\hline objsize $=3$ & .116 & .2719 & .181 & 1 & .671 & 1.123 & .659 & 1.913 \\
\hline $\begin{array}{l}\text { graspscen }=G \times \\
\text { objsize }=1\end{array}$ & -.415 & .3548 & 1.367 & 1 & .242 & .660 & .330 & 1.324 \\
\hline $\begin{array}{l}\text { graspscen }=G \times \\
\text { objsize }=2\end{array}$ & .078 & .3661 & .045 & 1 & .831 & 1.081 & .528 & 2.216 \\
\hline (Scale) & 1 & & & & & & & \\
\hline
\end{tabular}

3 


\section{Table 3 (on next page)}

Test of model effects from generalized estimating equation (GEE) models restricted to cases in which feet were observed contacting objects or substrates $(n=1503)$.

Toe configuration (toe code; $1=3 \times 1,2=2.5 \times 1.5,3=2 \times 2$ ) was modeled as a function of grasping scenario (graspscen; free-footed, grasping, perched), contact condition (contcond; F $=$ fish, $\mathrm{O}=$ other object, $\mathrm{T}=$ tree, $\mathrm{S}=$ other substrate) within grasping scenario, object size (objsize; small [1] - extra-large [4]), and footing (dual- or single-foot grasps). The reduced model shows results after excluding an interaction term with marginally non-significant parameter estimates; this model was further decomposed into separate models for each single $(n=541)$ and dual $(n=962)$ footing condition. 
1

\begin{tabular}{lccc}
\hline Source & Type III Wald $\chi^{2}$ & df & $\boldsymbol{P}$ \\
\hline \hline graspscen & 23.68 & 1 & $<.0001$ \\
objsize & 8.33 & 3 & .040 \\
footing & 5.20 & 1 & .023 \\
graspcond(graspscen) & 18.68 & 2 & $<.0001$ \\
footing $\times$ graspcond(graspscen) & 18.58 & 3 & .0003 \\
objsize $\times$ graspcond(graspscen) & 18.27 & 7 & .011 \\
& & & \\
Reduced model & & & \\
graspscen & 98.86 & 1 & $<.0001$ \\
objsize & 0.464 & 3 & .927 \\
footing & 5.25 & 1 & .022 \\
graspcond(graspscen) & 15.29 & 2 & $<.0001$ \\
footing $\times$ graspcond(graspscen) & 16.38 & 3 & .001 \\
& & & \\
Footing $=$ single-footed & & & \\
graspscen & 27.95 & 1 & $<.0001$ \\
objsize & .339 & 3 & .952 \\
graspcond(graspscen) & 18.30 & 2 & $<.0001$ \\
& & & \\
Footing $=$ dual-footed & & & \\
graspscen & 86.66 & 1 & $<.0001$ \\
objsize & .791 & 3 & .852 \\
graspcond(graspscen) & 1.92 & 2 & .383 \\
\hline
\end{tabular}

2 


\section{Table 4 (on next page)}

Parameter estimates and test statistics from generalized estimating equation (GEE) models for single-footed $(n=541)$ and dual-footed $(n=962)$ contact cases.

Toe configuration (toe code; $1=3 \times 1,2=2.5 \times 1.5,3=2 \times 2$ ) was modeled as a function of grasping scenario (graspscen; free-footed, grasping, perched), contact condition (contcond; F $=$ fish, $\mathrm{O}=$ other object, $\mathrm{T}=$ tree, $\mathrm{S}=$ other substrate) within grasping scenario, and object size (objsize; small [1] - extra-large [4]). 


\begin{tabular}{|c|c|c|c|c|c|c|c|c|}
\hline \multirow[b]{2}{*}{ Parameter } & \multirow[b]{2}{*}{$\boldsymbol{B}$} & \multirow[b]{2}{*}{$\begin{array}{l}\text { Std. } \\
\text { Error }\end{array}$} & \multicolumn{3}{|c|}{ Hypothesis Test } & \multirow[b]{2}{*}{$\begin{array}{c}\text { Odds ratio } \\
\operatorname{Exp}(B)\end{array}$} & \multicolumn{2}{|c|}{$\begin{array}{l}95 \% C I \\
\operatorname{Exp}(B) \\
\end{array}$} \\
\hline & & & $\begin{array}{l}\text { Type III } \\
\text { Wald } \chi^{2}\end{array}$ & df & $\boldsymbol{P}$ & & Lower & Upper \\
\hline \multicolumn{9}{|l|}{ Single-footed grasps } \\
\hline Threshold toecode $=1$ & .093 & .3531 & .070 & 1 & .792 & 1.098 & .549 & 2.193 \\
\hline toecode $=2$ & .731 & .3549 & 4.245 & 1 & .039 & 2.077 & 1.036 & 4.165 \\
\hline graspscen $=\mathrm{G}$ & 1.025 & .4664 & 4.826 & 1 & .028 & 2.786 & 1.117 & 6.950 \\
\hline graspscen $=\mathrm{P}$ & 0 & & & & & 1 & & \\
\hline objsize $=1$ & -.064 & .4638 & .019 & 1 & .890 & .938 & .378 & 2.327 \\
\hline objsize $=2$ & -.085 & .4461 & .036 & 1 & .850 & .919 & .383 & 2.203 \\
\hline objsize $=3$ & -.193 & .4261 & .204 & 1 & .651 & .825 & .358 & 1.902 \\
\hline objsize $=4$ & $0^{\mathrm{a}}$ & & & & & 1 & & \\
\hline contcond $=F($ graspscen $=G)$ & 1.400 & .3793 & 13.619 & 1 & .0002 & 4.054 & 1.928 & 8.527 \\
\hline contcond $=\mathrm{O}($ graspscen $=\mathrm{G})$ & $0^{\mathrm{a}}$ & & & & & 1 & & \\
\hline contcond $=\mathrm{T}($ graspscen $=\mathrm{P})$ & .666 & .3270 & 4.145 & 1 & .042 & 1.946 & 1.025 & 3.694 \\
\hline contcond $=\mathrm{S}($ graspscen $=\mathrm{P})$ & 0 & & & & & 1 & & \\
\hline (Scale) & 1 & & & & & & & \\
\hline \multicolumn{9}{|l|}{ Dual-footed grasps } \\
\hline Threshold toecode $=1$ & .373 & .3168 & 1.389 & 1 & .239 & 1.453 & .781 & 2.703 \\
\hline toecode $=2$ & 1.208 & .3208 & 14.188 & 1 & .0002 & 3.347 & 1.785 & 6.277 \\
\hline graspscen $=\mathrm{G}$ & 1.849 & .3149 & 34.456 & 1 & $<.0001$ & 6.352 & 3.426 & 11.775 \\
\hline graspscen $=P$ & 0 & & & & & 1 & & \\
\hline objsize $=1$ & .091 & .3589 & .064 & 1 & .800 & 1.095 & .542 & 2.213 \\
\hline objsize $=2$ & -.031 & .3538 & .007 & 1 & .931 & .970 & .485 & 1.940 \\
\hline objsize $=3$ & .122 & .3735 & .107 & 1 & .744 & 1.130 & .543 & 2.349 \\
\hline objsize $=4$ & 0 & & & & & 1 & & \\
\hline contcond $=F($ graspscen $=G)$ & -.142 & .2706 & .276 & 1 & .599 & .867 & .510 & 1.474 \\
\hline contcond $=\mathrm{O}($ graspscen $=\mathrm{G})$ & 0 & & & & & 1 & & \\
\hline contcond $=\mathrm{T}($ graspscen $=\mathrm{P})$ & .296 & .2257 & 1.722 & 1 & .189 & 1.345 & .864 & 2.093 \\
\hline contcond $=S($ graspscen $=P)$ & 0 & & & & & 1 & & \\
\hline (Scale) & 1 & & & & & & & \\
\hline
\end{tabular}

1 


\section{Figure 1}

Photos of Ospreys showing grasping scenarios and representative object types and sizes.

(A) Perched, grasping a tree branch (small) with a $2 \times 2$ configuration in the left foot and a $3 \times 1$ configuration in the right foot (photo by Scott Powell). (B) Perched, grasping a tree branch (small) with a $2.5 \times 1.5$ configuration in the left and right foot (photo by Scott Martin). (C) Perched, grasping a tree branch (medium) with a $3 \times 1$ configuration in the left foot and a $2.5 \times 1.5$ configuration in the right foot (photo by Peter J. Markham). (D) Perched, grasping (single-footed) a fish (large), with a $2 \times 2$ configuration in the left foot (photo by Emyr Evans/ Montgomeryshire Wildlife Trust). (E) Flying, grasping (dual-footed) a twig (small) using a $2 \times 2$ configuration in the left and right foot (photo by Tammy Karr). (F) Schematic diagrams of a left foot showing foot types scored in A-E. 

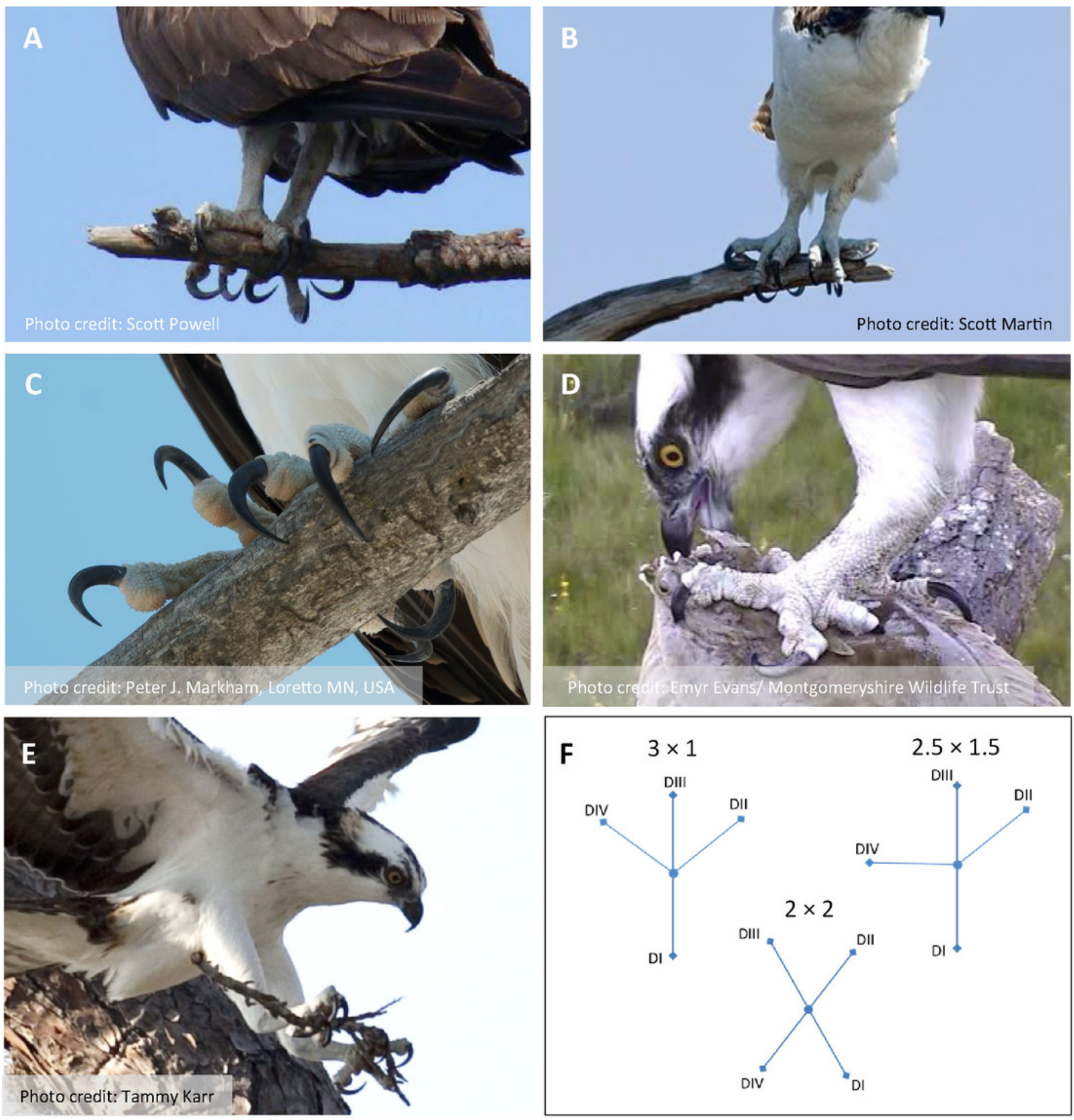


\section{Figure 2}

Raw proportional distributions of toe configurations with respect to grasping scenario and object size, scored from 1123 web images of Ospreys.

Toe configurations were classified as: $2 \times 2=$ zygodactyl, $3 \times 1=$ anisodactyl, and $2.5 \times 1.5=$ intermediate condition. The proportions of observations for each toe configuration across each grasping scenario (A), and relative object size class (B), were based on $n=1882$ feet (left and right combined). When these variables were considered in the analysis simultaneously, the probability of zygodactyly $(2 \times 2)$ was significantly greater when Ospreys were photographed grasping objects, or nothing, than when perched, and there was no significant effect of object size.

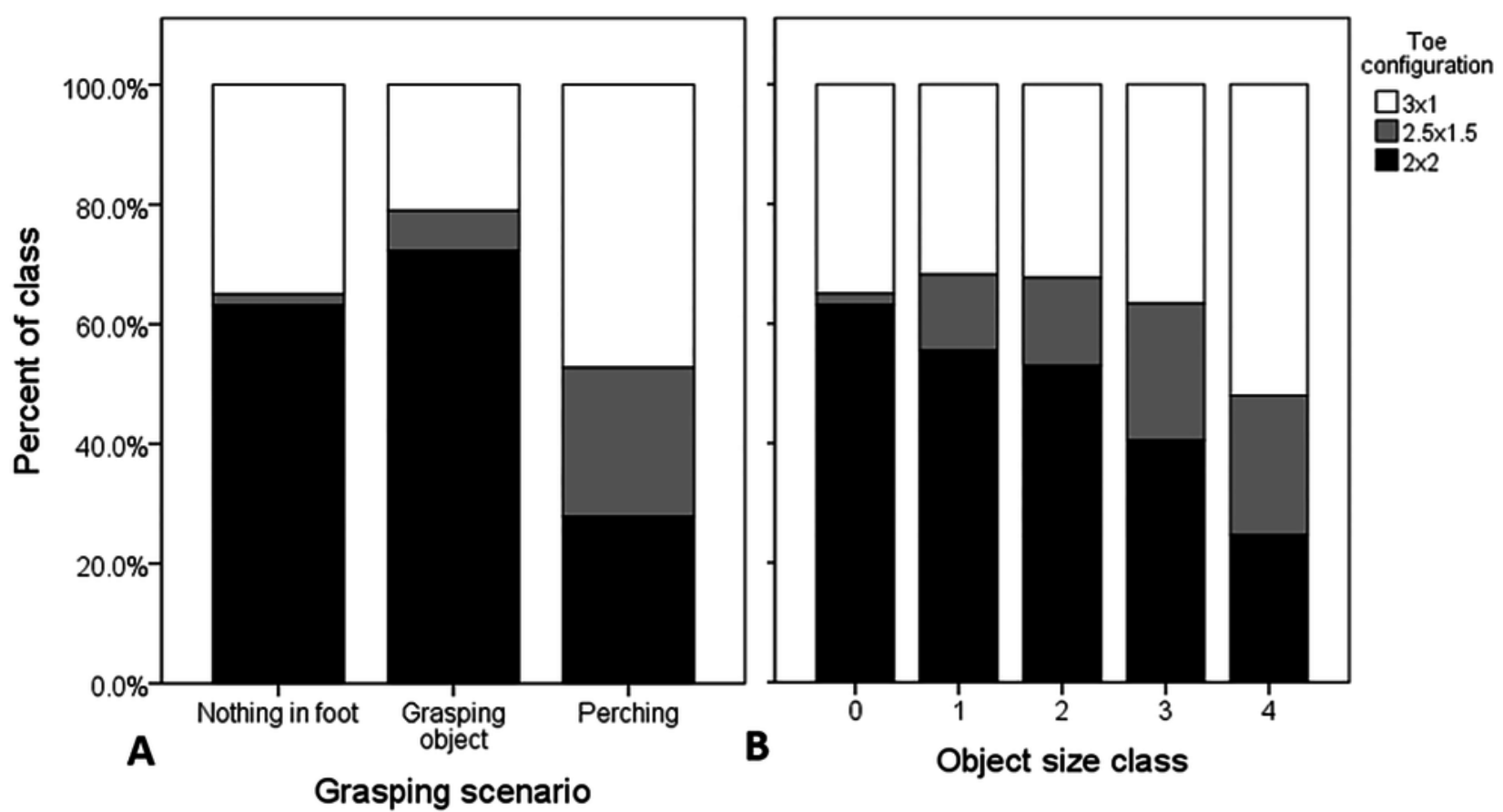




\section{Figure 3}

Raw proportional distributions of each toe configuration scored from 995 web images of Ospreys for single- and dual-foot grasps.

Toe configurations were classified as: $2 \times 2=$ zygodactyl, $3 \times 1=$ anisodactyl, and $2.5 \times 1.5=$ intermediate condition. Single-foot (A) and dual-foot (B) cross-tabulations with respect to grasping scenario and contact condition were based on $n=1503$ feet. When these variables were considered in the analysis simultaneously, the probability of zygodactyly $(2 \times 2)$ was significantly greater, overall, when Ospreys were photographed grasping compared to perching, and specifically for single-foot grasps of fish compared to other objects, and trees compared to other substrates.

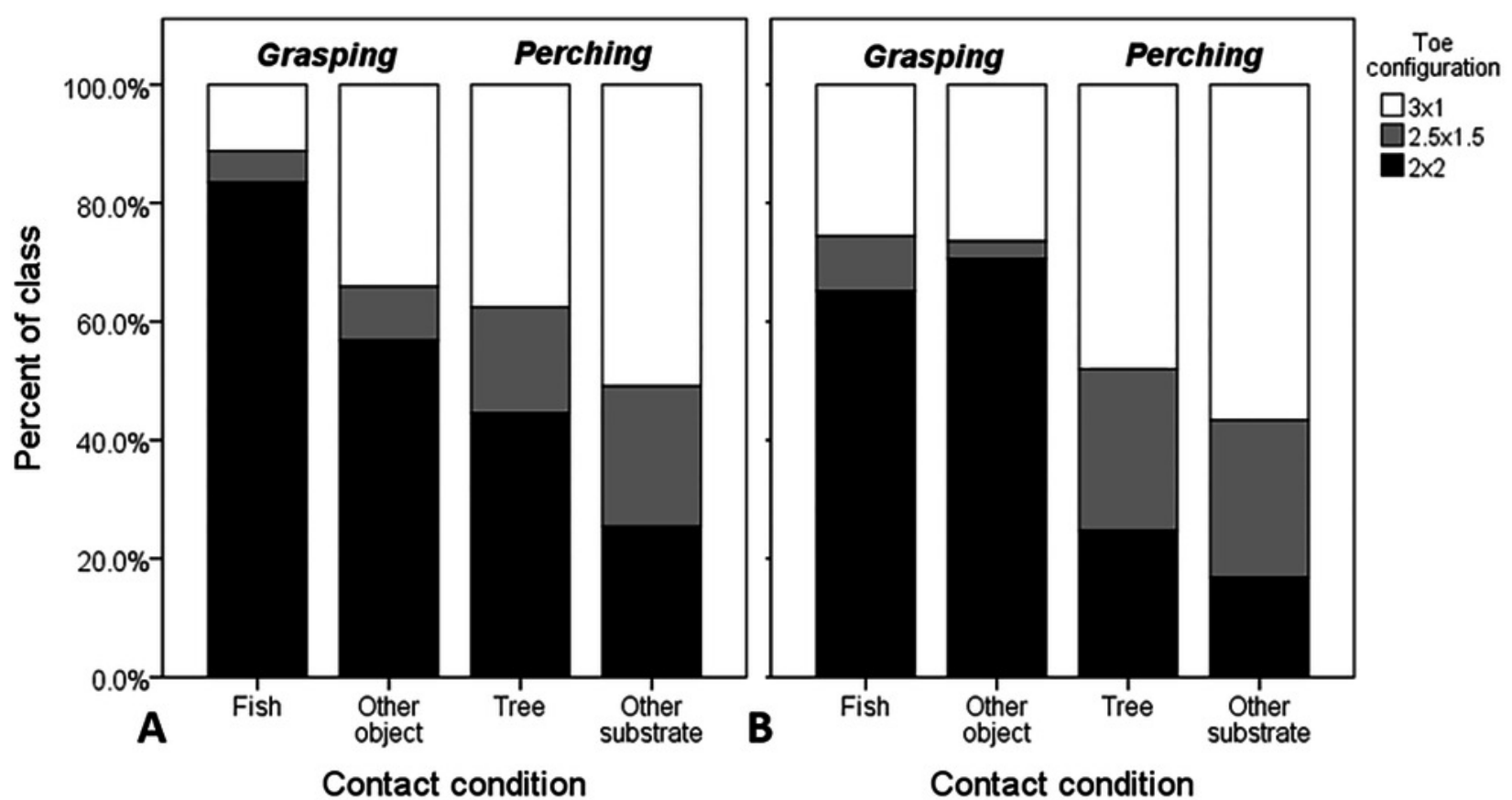

